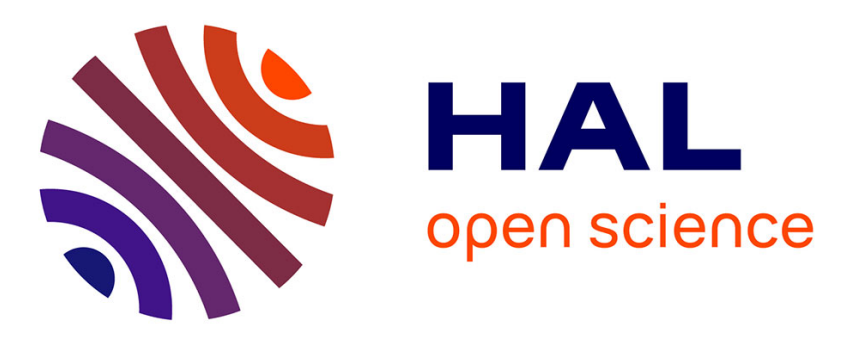

\title{
On model error in variational data assimilation
}

Victor Shutyaev, Arthur Vidard, François-Xavier Le Dimet, Igor Gejadze

\section{To cite this version:}

Victor Shutyaev, Arthur Vidard, François-Xavier Le Dimet, Igor Gejadze. On model error in variational data assimilation. Russian Journal of Numerical Analysis and Mathematical Modelling, 2016, 31 (2), pp.105-113. 10.1515/rnam-2016-0011 . hal-01309018

\section{HAL Id: hal-01309018 https://inria.hal.science/hal-01309018}

Submitted on 28 Apr 2016

HAL is a multi-disciplinary open access archive for the deposit and dissemination of scientific research documents, whether they are published or not. The documents may come from teaching and research institutions in France or abroad, or from public or private research centers.
L'archive ouverte pluridisciplinaire HAL, est destinée au dépôt et à la diffusion de documents scientifiques de niveau recherche, publiés ou non, émanant des établissements d'enseignement et de recherche français ou étrangers, des laboratoires publics ou privés. 


\title{
On model error in variational data assimilation
}

\author{
Victor Shutyaev ${ }^{1}$, Arthur Vidard ${ }^{2}$, Francois-Xavier Le Dimet ${ }^{2}$, and Igor Gejadze ${ }^{3}$
}

\begin{abstract}
The problem of variational data assimilation for a nonlinear evolution model is formulated as an optimal control problem to find the initial condition. The optimal solution (analysis) error arises due to the errors in the input data (background and observation errors). Under the gaussian assumption the optimal solution error covariance can be constructed using the Hessian of the auxiliary data assimilation problem. The aim of this paper is to study the evolution of model errors via data assimilation. The optimal solution error covariances are derived in the case of imperfect model and for the weak constraint formulation, when the model equations determine the cost functional.
\end{abstract}

Keywords: data assimilation, optimal control, tangent linear approximation, analysis error covariance, model error

MSC: $65 \mathrm{~K} 10$

\section{Introduction}

One of the main limitation of the current operational variational data assimilation techniques is that they assume the model to be perfect mainly because of computing cost issues. In conventional strong constraint variational data assimilation involving the global-scale atmospheric and ocean models the initial condition of the dynamical system is considered as control. In doing so one believes that the state evolution is largely defined by its initial value, which is only true to a certain time extent. This is the main motivation for considering the weak constraint variational data assimilation (Sasaki, 1970), where the model error is regarded as a forcing term to be controlled.

Numerous researches have been carried out to reduce the cost of controlling model errors by controlling the correction term only in certain privileged directions or by controlling only the systematic and time correlated part of the error (Vidard, 2001, 2004; Griffith and Nichols, 1996, 2000; Akella and Navon, 2009, and oths.).

A reduction of the systematic model error control vector size by projecting it on to the subspace of eigenvectors corresponding to the leading eigenvalues of the adjoint-tangent linear operators was illustrated by Vidard (2001, 2000). Vidard et al. (2004) considered a continuousin-time form for the evolution of the model error. This approach is consistent with the fact that model equations are first written as continuous differential equations and then descretized in space and time. Such a deterministic approach to model the evolution of the model error significantly simplifies the weak constraint variational data assimilation since only the initial model error is to be obtained via solution of the optimization problem.

\footnotetext{
${ }^{1}$ Institute of Numerical Mathematics, Russian Academy of Sciences, Moscow Institute for Physics and Technology, 119333 Gubkina 8, Moscow, Russia. E-mail: shutyaev@inm.ras.ru

${ }^{2}$ LJK, Université de Grenoble Alpes, BP 51, 38051 Grenoble Cedex 9, France

${ }^{3}$ UMR G-EAU, IRSTEA-Montpellier, 361 Rue J.F. Breton, BP 5095, 34196, Montpellier, France
} 
A consistent method of decreasing the discretization errors (principally truncation errors) is to refine the model resolution, such an approach in variational data assimilation was studied by Le Dimet and Shutyaev (2005). The control of the discretization error as the only source of the model error was considered by Le Dimet et al. (2008).

The above methods consider the model errors as a forcing term in the model equations. Trémolet (2006) describes another approach where the full state vector (4D field: 3D spatial + time) is controlled. Because of computing cost one cannot obviously control the model state at each time step. Therefore, the assimilation window is split into sub-windows, and only the initial conditions of each sub-window are controlled, the junctions between each sub-window being penalized. One interesting property is that, in this case, the computation of the gradients, for the different sub-windows, are independent and therefore can be done in parallel.

Shutyaev, Le Dimet and Gejadze (2006, 2009) derived the equation for the error of the optimal solution through the statistical errors of the input data (background, observation, and model errors). The numerical algorithm is developed to construct the analysis error covariance operator of the using the Hessian of an auxiliary optimal control problem based on the tangent linear model constraints. Some basic error analysis for the weak constraint variational data assimilation is given in earlier paper by Gejadze and Shutyaev (1999).

In this paper we try to combine the approaches developed by Vidard et al. (2004) and Shutyaev et al. (2009) and to study the error covariances in the weak constraint variational data assimilation with the model error governed by an evolution equation, and with the cost functional involving the initial model error covariance matrix. Computing the optimal solution error covariances is based on the inverse Hessian of the auxiliary control problem.

\section{Data assimilation problem for perfect model}

Consider the mathematical model of a physical process that is described by the evolution problem:

$$
\left\{\begin{aligned}
\frac{\partial \varphi}{\partial t} & =\mathcal{F}(\varphi), \quad t \in(0, T) \\
\left.\varphi\right|_{t=0} & =u
\end{aligned}\right.
$$

where $\varphi=\varphi(t)$ is the unknown function belonging for any $t \in(0, T)$ to a state space $X, u \in X$, $\mathcal{F}$ is a nonlinear operator mapping $X$ into $X$. Let $Y=L_{2}(0, T ; X)$ be a space of functions $\varphi(t)$ with values in $X,\|\cdot\|_{Y}=(\cdot, \cdot)_{Y}^{1 / 2}$. Suppose that for a given $u \in X$ there exists a unique solution $\varphi \in Y$ to $(1.1)$.

First we accept the 'perfect model' assumption, i.e. $\mathcal{F}$ is known without error.

Let $\bar{u}$ be the "true" initial state and $\bar{\varphi}$ - the solution to the problem (1.1) with $u=\bar{u}$, i.e. the "true" state evolution:

$$
\left\{\begin{aligned}
\frac{\partial \bar{\varphi}}{\partial t} & =\mathcal{F}(\bar{\varphi}), \quad t \in(0, T) \\
\left.\bar{\varphi}\right|_{t=0} & =\bar{u} .
\end{aligned}\right.
$$

We define the input data as follows: the background function $u_{b} \in X, u_{b}=\bar{u}+\xi_{b}$ and the observations $y \in Y_{o}, y=C \bar{\varphi}+\xi_{o}$, where $C: Y \rightarrow Y_{o}$ is a linear bounded operator (observation operator) and $Y_{o}$ is an observation space.

The functions $\xi_{b} \in X$ and $\xi_{o} \in Y_{o}$ may be regarded as the background and the observation error, respectively. We assume that these errors are normally distributed (Gaussian) with 
zero mean and the covariance operators $V_{b} \cdot=E\left[\left(\cdot, \xi_{b}\right)_{X} \xi_{b}\right]$ and $V_{o} \cdot=E\left[\left(\cdot, \xi_{o}\right)_{Y_{o}} \xi_{o}\right]$, i.e. $\xi_{b} \sim \mathcal{N}\left(0, V_{b}\right), \xi_{o} \sim \mathcal{N}\left(0, V_{o}\right)$, where " $\sim$ " is read "is distributed as". We also assume that $\xi_{o}$, $\xi_{b}$ are mutually uncorrelated and $V_{b}, V_{o}$ are positive definite, hence invertible.

Let us formulate the following data assimilation problem (optimal control problem) with the aim to identify the initial condition: for given $u_{b} \in X, y \in Y_{o}$ find $u \in X$ and $\varphi \in Y$ such that they satisfy (1.1), and on the set of solutions to (1.1), a cost functional $J$ takes the minimum value, i.e.

$$
J(u)=\inf _{v \in X} J(v)
$$

where

$$
J(u)=\frac{1}{2}\left(V_{b}^{-1}\left(u-u_{b}\right), u-u_{b}\right)_{X}+\frac{1}{2}\left(V_{o}^{-1}(C \varphi-y), C \varphi-y\right)_{Y_{o}} .
$$

The necessary optimality condition reduces the problem (1.3)-(1.4) to the optimality system [14]:

$$
\begin{gathered}
\left\{\begin{aligned}
\frac{\partial \varphi}{\partial t} & =\mathcal{F}(\varphi), \quad t \in(0, T) \\
\left.\varphi\right|_{t=0} & =u,
\end{aligned}\right. \\
\left\{\begin{aligned}
-\frac{\partial \varphi^{*}}{\partial t}-\left(\mathcal{F}^{\prime}(\varphi)\right)^{*} \varphi^{*} & =-C^{*} V_{o}^{-1}(C \varphi-y), \quad t \in(0, T) \\
\left.\varphi^{*}\right|_{t=T} & =0, \\
V_{b}^{-1}(u & \left.-u_{b}\right)-\left.\varphi^{*}\right|_{t=0}=0
\end{aligned}\right.
\end{gathered}
$$

with the unknowns $\varphi, \varphi^{*}, u$, where $\left(\mathcal{F}^{\prime}(\varphi)\right)^{*}$ is the adjoint to the Frechet derivative of $\mathcal{F}$, and $C^{*}$ is the adjoint to $C$ defined by $(C \varphi, \psi)_{Y_{o}}=\left(\varphi, C^{*} \psi\right)_{Y}, \varphi \in Y, \psi \in Y_{o}$.

We define the error of the optimal solution as $\delta u=u-\bar{u}$. Under the assumption of tangent liner hypothethis (TLH) the equation for $\delta u$ is valid [4]:

$$
\mathcal{H} \delta u=R_{1} \xi_{b}+R_{2} \xi_{o},
$$

where $\mathcal{H}$ is the Hessian of the auxiliary data assimilation problem defined successively by the equations

$$
\begin{gathered}
\left\{\begin{array}{c}
\frac{\partial \delta \varphi}{\partial t}=\mathcal{F}^{\prime}(\bar{\varphi}) \delta \varphi, t \in(0, T) \\
\left.\delta \varphi\right|_{t=0}=\delta u,
\end{array}\right. \\
\left\{\begin{array}{c}
-\frac{\partial \varphi^{*}}{\partial t}-\left(\mathcal{F}^{\prime}(\bar{\varphi})\right)^{*} \varphi^{*}=-C^{*} V_{o}^{-1} C \delta \varphi, \quad t \in(0, T) \\
\left.\varphi^{*}\right|_{t=T}=0, \\
\mathcal{H} \delta u=V_{b}^{-1} \delta u-\left.\varphi^{*}\right|_{t=0},
\end{array}\right.
\end{gathered}
$$

and the operators $R_{1}: X \rightarrow X, R_{2}: Y_{o} \rightarrow X$ are defined by

$$
R_{1}=V_{b}^{-1}, R_{2} g=\left.\theta^{*}\right|_{t=0}
$$

with $\theta^{*}$ being the solution to

$$
\left\{\begin{aligned}
-\frac{\partial \theta^{*}}{\partial t}-\left(\mathcal{F}^{\prime}(\bar{\varphi})\right)^{*} \theta^{*} & =C^{*} V_{o}^{-1} g, \quad t \in(0, T) \\
\left.\theta^{*}\right|_{t=T} & =0 .
\end{aligned}\right.
$$

From (1.8) it follows [4] that the optimal solution error covariance $V_{\delta u} \cdot=E\left[(\cdot, \delta u)_{X} \delta u\right]$ is the inverse Hessian:

$$
V_{\delta u}=\mathcal{H}^{-1}
$$




\section{Data assimilation problem for imperfect model}

Instead of (1.1) let us consider the imperfect model

$$
\left\{\begin{aligned}
\frac{\partial \varphi}{\partial t} & =F(\varphi), \quad t \in(0, T) \\
\left.\varphi\right|_{t=0} & =u
\end{aligned}\right.
$$

with some operator $F$ mapping $X$ into $X$ which is supposed to be some approximation of the exact operator $\mathcal{F}$. However, the true solution $\bar{\varphi}$ is still defined by the perfect model (1.2). We rewrite (1.2) in the form

$$
\left\{\begin{aligned}
\frac{\partial \bar{\varphi}}{\partial t} & =F(\bar{\varphi})+\bar{f}, \quad t \in(0, T) \\
\left.\bar{\varphi}\right|_{t=0} & =\bar{u}
\end{aligned}\right.
$$

where

$$
\bar{f}=\mathcal{F}(\bar{\varphi})-F(\bar{\varphi})
$$

and suppose that the model error $\bar{f}$ satisfies the evolution problem

$$
\left\{\begin{aligned}
\frac{\partial \bar{f}}{\partial t} & =\Phi(\bar{\varphi}) \bar{f}, \quad t \in(0, T) \\
\left.\bar{f}\right|_{t=0} & =\xi_{m}
\end{aligned}\right.
$$

where $\Phi(\bar{\varphi}): Y \rightarrow Y$ is linear operator depending on $\bar{\varphi}$, and $\xi_{m}$ is the initial model error. We assume that $\xi_{m} \sim \mathcal{N}\left(0, V_{m}\right)$ with some positive definite covariance operator $V_{m}$.

We define the input data $u_{b}$ and $y$ as in Section 1 , and formulate the following data assimilation problem: for given $u_{b} \in X, y \in Y_{o}$ find $u \in X$ and $\varphi \in Y$ such that

$$
\left\{\begin{aligned}
\frac{\partial \varphi}{\partial t} & =F(\varphi), \quad t \in(0, T) \\
\left.\varphi\right|_{t=0} & =u \\
J(u) & =\inf _{v \in X} J(v),
\end{aligned}\right.
$$

with the cost function $J$ defined by (1.4).

To derive the equation for the optimal solution error $\delta u=u-\bar{u}$, we consider the optimality system related to (2.4) and the perfect model (2.2). For small errors $\delta \varphi=\varphi-\bar{\varphi}$ we get

$$
\begin{gathered}
\left\{\begin{aligned}
\frac{\partial \delta \varphi}{\partial t}= & F^{\prime}(\bar{\varphi}) \delta \varphi-\bar{f}, \quad t \in(0, T) \\
\left.\delta \varphi\right|_{t=0}= & \delta u
\end{aligned}\right. \\
\left\{\begin{array}{c}
-\frac{\partial \varphi^{*}}{\partial t}-\left(F^{\prime}(\bar{\varphi})\right)^{*} \varphi^{*}=-C^{*} V_{o}^{-1}\left(C \delta \varphi-\xi_{o}\right), \quad t \in(0, T) \\
\left.\varphi^{*}\right|_{t=T}=0, \\
V_{b}^{-1}\left(\delta u-\xi_{b}\right)-\left.\varphi^{*}\right|_{t=0}=0
\end{array}\right.
\end{gathered}
$$

with $\bar{f}$ defined by (2.3). From $(2.5)-(2.7)$ we obtain the error equation

$$
H \delta u=R_{1} \xi_{b}+R_{2} \xi_{o}+R_{3} \xi_{m}
$$


where $H$ is the Hessian of the auxiliary data assimilation problem defined by the equations

$$
\begin{gathered}
\left\{\begin{array}{c}
\frac{\partial \psi}{\partial t}=F^{\prime}(\bar{\varphi}) \psi, t \in(0, T) \\
\left.\psi\right|_{t=0}=v,
\end{array}\right. \\
\left\{\begin{array}{c}
-\frac{\partial \varphi^{*}}{\partial t}-\left(F^{\prime}(\bar{\varphi})\right)^{*} \varphi^{*}=-C^{*} V_{o}^{-1} C \psi, t \in(0, T) \\
\left.\varphi^{*}\right|_{t=T}=0, \\
H v=V_{b}^{-1} v-\left.\varphi^{*}\right|_{t=0},
\end{array}\right.
\end{gathered}
$$

the operators $R_{1}: X \rightarrow X, R_{2}: Y_{o} \rightarrow X$ are defined by

$$
R_{1}=V_{b}^{-1}, R_{2} g=\left.\eta^{*}\right|_{t=0}
$$

with $\eta^{*}$ being the solution to

$$
\left\{\begin{aligned}
-\frac{\partial \eta^{*}}{\partial t}-\left(F^{\prime}(\bar{\varphi})\right)^{*} \eta^{*} & =C^{*} V_{o}^{-1} g, \quad t \in(0, T) \\
\left.\eta^{*}\right|_{t=T} & =0
\end{aligned}\right.
$$

and the operator $R_{3}: X \rightarrow X$ is defined successively as

$$
\begin{gathered}
\left\{\begin{aligned}
\frac{\partial \psi}{\partial t} & =\Phi(\bar{\varphi}) \psi, t \in(0, T) \\
\left.\psi\right|_{t=0} & =h,
\end{aligned}\right. \\
\left\{\begin{aligned}
\frac{\partial \theta}{\partial t}= & F^{\prime}(\bar{\varphi}) \theta+\psi, \quad t \in(0, T) \\
\left.\theta\right|_{t=0}= & 0,
\end{aligned}\right. \\
\left\{\begin{aligned}
-\frac{\partial \theta^{*}}{\partial t}-\left(F^{\prime}(\bar{\varphi})\right)^{*} \theta^{*} & =-C^{*} V_{o}^{-1} C \theta, \quad t \in(0, T) \\
\left.\theta^{*}\right|_{t=T} & =0, \\
R_{3} h & =-\left.\theta^{*}\right|_{t=0} .
\end{aligned}\right.
\end{gathered}
$$

From (2.8) it follows that the optimal solution error covariance $V_{\delta u}$ is

$$
V_{\delta u}=H^{-1}+H^{-1} R_{3} V_{m} R_{3}^{*} H^{-1} .
$$

Here the adjoint operator $R_{3}^{*}$ is defined by the equations

$$
\begin{gathered}
\left\{\begin{array}{r}
\frac{\partial \phi}{\partial t}=F^{\prime}(\bar{\varphi}) \phi, \quad t \in(0, T) \\
\left.\phi\right|_{t=0}=p
\end{array}\right. \\
\left\{\begin{array}{r}
-\frac{\partial \phi^{*}}{\partial t}-\left(F^{\prime}(\bar{\varphi})\right)^{*} \phi^{*}=-C^{*} V_{o}^{-1} C \phi, \quad t \in(0, T) \\
\left.\phi^{*}\right|_{t=T}=0,
\end{array}\right. \\
\left\{\begin{aligned}
&-\frac{\partial \psi^{*}}{\partial t}= \Phi^{*}(\bar{\varphi}) \psi^{*}+\phi^{*}, \quad t \in(0, T) \\
&\left.\psi^{*}\right|_{t=T}= 0, \\
& R_{3}^{*} p=-\left.\psi^{*}\right|_{t=0} .
\end{aligned}\right.
\end{gathered}
$$


The operator $R_{3} V_{m} R_{3}^{*}$ involved in (2.18) may be defined successively by

$$
\begin{gathered}
\left\{\begin{array}{c}
\frac{\partial \phi}{\partial t}=F^{\prime}(\bar{\varphi}) \phi, \quad t \in(0, T) \\
\left.\phi\right|_{t=0}=p
\end{array}\right. \\
\left\{\begin{array}{c}
-\frac{\partial \phi^{*}}{\partial t}-\left(F^{\prime}(\bar{\varphi})\right)^{*} \phi^{*}=-C^{*} V_{o}^{-1} C \phi, \quad t \in(0, T) \\
\left.\phi^{*}\right|_{t=T}=0,
\end{array}\right. \\
\left\{\begin{array}{c}
-\frac{\partial \psi^{*}}{\partial t}=\Phi^{*}(\bar{\varphi}) \psi^{*}+\phi^{*}, \quad t \in(0, T) \\
\left.\psi^{*}\right|_{t=T}=0,
\end{array}\right. \\
\left\{\begin{array}{c}
\frac{\partial \psi}{\partial t}=\Phi(\bar{\varphi}) \psi, t \in(0, T) \\
\left.\psi\right|_{t=0}=-\left.V_{m} \psi^{*}\right|_{t=0}, \\
\frac{\partial \theta}{\partial t}=F^{\prime}(\bar{\varphi}) \theta+\psi, \quad t \in(0, T) \\
\left.\theta\right|_{t=0}=0, \\
\left\{\frac{\partial \theta^{*}}{\partial t}-\left(F^{\prime}(\bar{\varphi})\right)^{*} \theta^{*}=-C^{*} V_{o}^{-1} C \theta, t \in(0, T)\right. \\
\left.\theta^{*}\right|_{t=T}=0, \\
R_{3} V_{m} R_{3}^{*} p=-\left.\theta^{*}\right|_{t=0} .
\end{array}\right.
\end{gathered}
$$

Let us note that the system of equations (2.24)-(2.25) is adjoint with respect to the system of equations (2.26)-(2.27).

\section{Data assimilation problem for extended imperfect model}

In this section, instead of (2.1) we consider the extended imperfect model

$$
\left\{\begin{aligned}
\frac{\partial \varphi}{\partial t} & =F(\varphi)+f, \quad t \in(0, T) \\
\left.\varphi\right|_{t=0} & =u
\end{aligned}\right.
$$

with the unknown model error $f$ which is supposed to satisfy the evolution problem

$$
\left\{\begin{aligned}
\frac{\partial f}{\partial t} & =\Phi(\varphi) f, \quad t \in(0, T) \\
\left.f\right|_{t=0} & =f_{0}
\end{aligned}\right.
$$

where $\Phi(\varphi): Y \rightarrow Y$ is linear operator introduced above depending on $\varphi$, and $f_{0}$ is the initial model error.

We assume again that the true solution $\bar{\varphi}$ is given by $(2.2)-(2.3)$, and consider the same input data $u_{b}$ and $y$ defined by the true solution as above.

Now we formulate the following data assimilation problem: for given $u_{b} \in X, y \in Y_{o}$ find $u, f_{0} \in X$ and $\varphi \in Y$ such that they satisfy (3.1)-(3.2), and on the set of solutions to (3.1)-(3.2), a cost functional $J_{1}$ takes the minimum value, i.e.

$$
J_{1}\left(u, f_{0}\right)=\inf _{v, g \in X} J_{1}(v, g)
$$


where

$$
J_{1}\left(u, f_{0}\right)=\frac{1}{2}\left(V_{b}^{-1}\left(u-u_{b}\right), u-u_{b}\right)_{X}+\frac{1}{2}\left(V_{o}^{-1}(C \varphi-y), C \varphi-y\right)_{Y_{o}}+\frac{1}{2}\left(V_{m}^{-1} f_{0}, f_{0}\right)_{X}
$$

with the covariance operator $V_{m}$ defined in Section 2 .

The optimality system has the form

$$
\begin{aligned}
& \left\{\begin{aligned}
\frac{\partial \varphi}{\partial t} & =F(\varphi)+f, \quad t \in(0, T) \\
\left.\varphi\right|_{t=0} & =u
\end{aligned}\right. \\
& \left\{\begin{aligned}
\frac{\partial f}{\partial t} & =\Phi(\varphi) f, \quad t \in(0, T) \\
\left.f\right|_{t=0} & =f_{0}
\end{aligned}\right. \\
& \left\{\begin{aligned}
-\frac{\partial \varphi^{*}}{\partial t}-\left(F^{\prime}(\varphi)\right)^{*} \varphi^{*}-\left(\Phi^{\prime}(\varphi) \cdot f\right)^{*} f^{*} & =-C^{*} V_{o}^{-1}(C \varphi-y), \quad t \in(0, T) \\
\left.\varphi^{*}\right|_{t=T} & =0
\end{aligned}\right. \\
& \left\{\begin{aligned}
-\frac{\partial f^{*}}{\partial t}-(\Phi(\varphi))^{*} f^{*} & =\varphi^{*}, \quad t \in(0, T) \\
\left.f^{*}\right|_{t=T} & =0
\end{aligned}\right. \\
& V_{b}^{-1}\left(u-u_{b}\right)-\left.\varphi^{*}\right|_{t=0}=0 \text {, } \\
& V_{m}^{-1} f_{0}-\left.f^{*}\right|_{t=0}=0 .
\end{aligned}
$$

Under the TLH assumption, from (3.5)-(3.10) and (2.2)-(2.3) we obtain the system for the errors $\delta \varphi=\varphi-\bar{\varphi}, \delta f=f-\bar{f}, \delta u=u-\bar{u}$ :

$$
\begin{aligned}
& \left\{\begin{aligned}
\frac{\partial \delta \varphi}{\partial t} & =F^{\prime}(\bar{\varphi}) \delta \varphi+\delta f, \quad t \in(0, T) \\
\left.\delta \varphi\right|_{t=0} & =\delta u
\end{aligned}\right. \\
& \left\{\begin{aligned}
\frac{\partial \delta f}{\partial t} & =\Phi(\bar{\varphi}) \delta f+\Phi^{\prime}(\bar{\varphi}) \delta \varphi \bar{f}, \quad t \in(0, T) \\
\left.\delta f\right|_{t=0} & =f_{0}-\xi_{m}
\end{aligned}\right. \\
& \left\{\begin{aligned}
-\frac{\partial \varphi^{*}}{\partial t}-\left(F^{\prime}(\bar{\varphi})\right)^{*} \varphi^{*}-\left(\Phi^{\prime}(\bar{\varphi}) \cdot \bar{f}\right)^{*} f^{*} & =-C^{*} V_{o}^{-1}\left(C \delta \varphi-\xi_{o}\right), \quad t \in(0, T) \\
\left.\varphi^{*}\right|_{t=T} & =0,
\end{aligned}\right. \\
& \left\{\begin{aligned}
-\frac{\partial f^{*}}{\partial t}-(\Phi(\bar{\varphi}))^{*} f^{*} & =\varphi^{*}, \quad t \in(0, T) \\
\left.f^{*}\right|_{t=T} & =0
\end{aligned}\right. \\
& V_{b}^{-1}\left(\delta u-\xi_{b}\right)-\left.\varphi^{*}\right|_{t=0}=0, \\
& \left.V_{m}^{-1} \delta f\right|_{t=0}-\left.f^{*}\right|_{t=0}=-V_{m}^{-1} \xi_{m} .
\end{aligned}
$$

The system (3.11)-(3.16) is equivalent to the following data assimilation problem: find $\delta u, \delta f_{0} \in X$ such that

$$
\left\{\begin{aligned}
\frac{\partial \delta \varphi}{\partial t} & =F^{\prime}(\bar{\varphi}) \delta \varphi+\delta f, \quad t \in(0, T) \\
\left.\delta \varphi\right|_{t=0} & =\delta u
\end{aligned}\right.
$$




$$
\left\{\begin{aligned}
\frac{\partial \delta f}{\partial t} & =\Phi(\bar{\varphi}) \delta f+\Phi^{\prime}(\bar{\varphi}) f \delta \varphi, \quad t \in(0, T) \\
\left.\delta f\right|_{t=0} & =\delta f_{0}, \\
& J_{2}\left(\delta u, \delta f_{0}\right)=\inf _{v, g \in X} J_{2}(v, g),
\end{aligned}\right.
$$

where

$J_{2}\left(\delta u, \delta f_{0}\right)=\frac{1}{2}\left(V_{b}^{-1}\left(\delta u-\xi_{b}\right), \delta u-\xi_{b}\right)_{X}+\frac{1}{2}\left(V_{o}^{-1}\left(C \delta \varphi-\xi_{o}\right), C \delta \varphi-\xi_{o}\right)_{Y_{o}}+\frac{1}{2}\left(V_{m}^{-1}\left(\delta f_{0}+\xi_{m}\right), \delta f_{0}+\xi_{m}\right)_{X}$

with the covariance operator $V_{m}$ defined above.

The linear data assimilation problem (3.17)-(3.19) is equivalent to a single equation for the control variables:

$$
\mathbf{H} \delta U=R_{1} \Xi+R_{2} \xi_{o},
$$

where $\mathbf{H}$ is the Hessian of the functional (3.20), $\delta U=\left(\delta u, \delta f_{0}\right)^{T}, \Xi=\left(\xi_{b}, \xi_{m}\right)^{T}, R_{1} \Xi=$ $\left(V_{b}^{-1} \xi_{b},-V_{m}^{-1} \xi_{m}\right)^{T}, R_{2} \xi_{o}=\left(\left.\tilde{\varphi}^{*}\right|_{t=0},\left.\tilde{f}^{*}\right|_{t=0}\right)^{T}$, with $\tilde{\varphi}^{*}, \tilde{f}^{*}$ satysfying the system

$$
\begin{aligned}
& \left\{\begin{aligned}
-\frac{\partial \tilde{\varphi}^{*}}{\partial t}-\left(F^{\prime}(\bar{\varphi})\right)^{*} \tilde{\varphi}^{*}-\left(\Phi^{\prime}(\bar{\varphi}) \cdot \bar{f}\right)^{*} \tilde{f}^{*} & =C^{*} V_{o}^{-1} C \xi_{o}, \quad t \in(0, T) \\
\left.\tilde{\varphi}^{*}\right|_{t=T} & =0
\end{aligned}\right. \\
& \left\{\begin{aligned}
-\frac{\partial \tilde{f}^{*}}{\partial t}-(\Phi(\bar{\varphi}))^{*} \tilde{f}^{*} & =\tilde{\varphi}^{*}, \quad t \in(0, T) \\
\left.\tilde{f}^{*}\right|_{t=T} & =0 .
\end{aligned}\right.
\end{aligned}
$$

The Hessian $\mathbf{H}$ is defined on pairs $V=(v, w)^{T}$ by the successive solutions of the following problems:

$$
\begin{gathered}
\left\{\begin{array}{r}
\frac{\partial \psi}{\partial t}=F^{\prime}(\bar{\varphi}) \psi+\eta, \quad t \in(0, T) \\
\left.\psi\right|_{t=0}=v,
\end{array}\right. \\
\left\{\begin{array}{r}
\frac{\partial \eta}{\partial t}=\Phi(\bar{\varphi}) \eta+\Phi^{\prime}(\bar{\varphi}) \psi \bar{f}, \quad t \in(0, T) \\
\left.\eta\right|_{t=0}=w,
\end{array}\right. \\
\left\{\begin{array}{r}
-\frac{\partial \psi^{*}}{\partial t}-\left(F^{\prime}(\bar{\varphi})\right)^{*} \psi^{*}-\left(\Phi^{\prime}(\bar{\varphi}) \cdot \bar{f}\right)^{*} \eta^{*}=-C^{*} V_{o}^{-1} C \psi, \quad t \in(0, T) \\
\left.\psi^{*}\right|_{t=T}=0,
\end{array}\right. \\
\left\{\begin{array}{r}
-\frac{\partial \eta^{*}}{\partial t}-(\Phi(\bar{\varphi}))^{*} \eta^{*}= \\
\left.\eta^{*}\right|_{t=T}=0,
\end{array}\right. \\
\mathbf{H} V=\left(V_{b}^{-1} v-\left.\psi^{*}\right|_{t=0}, V_{m}^{-1} w-\left.\eta^{*}\right|_{t=0}\right)^{T} .
\end{gathered}
$$

Following [4], we get from (3.21) that the optimal solution error covariance $V_{\delta U}$ is the inverse Hessian:

$$
V_{\delta U}=\mathbf{H}^{-1}
$$

The formulas (2.18) and (3.29) can be used to quantify the optimal solution error via data assimilation for imperfect models. 


\section{Acknowledgments}

This work was carried out within the SAMOVAR project (CNRS-RAS), Russian Science Foundation project 14-11-00609 (studies in Section 3), and the project 15-01-01583 of the Russian Foundation for the Basic Research.

\section{References}

[1] Akella S., Navon I.M. Different approaches to model error formulation in 4D-Var: a study with high resolution advection schemes. Tellus, 2009, v.61A, pp. 112-128.

[2] Fisher M., Courtier P. Estimating the covariance matrices of analysis and forecast error in variational data assimilation. ECMWF Research Department Techn. Memo. 220, 1995.

[3] Furbish D., Hussaini M. Y., Le Dimet F.-X., Ngnepieba P., and Wu Y. On discretization error and its control in variational data assimilation. Tellus, 2008, v.60A, pp.979-991.

[4] Gejadze I., Le Dimet F.-X., Shutyaev V. On analysis error covariances in variational data assimilation. SIAM J. Sci. Computing, 2008, v.30, no.4, pp.1847-1874.

[5] Gejadze I., Le Dimet F.-X., Shutyaev V. On optimal solution error covariances in variational data assimilation problems. Journal of Computational Physics, 2010, v.229, pp.21592178 .

[6] Gejadze I., Le Dimet F.-X., Shutyaev V. Computation of the optimal solution error covariance in variational data assimilation problems with nonlinear dynamics. Journal of Computational Physics, 2011, v.230, pp.79-7943.

[7] Gejadze I., Shutyaev V. An optimal control problem of initial data restoration. Computational Mathematics and Mathematical Physics, 1999, v.39, no.9, pp.1416-1425.

[8] Gejadze I., Shutyaev V., Le Dimet F.-X. Analysis error covariance versus posterior covariance in variational data assimilation. Q.J.R. Meteorol. Soc., 2012, v.138, pp.1-16.

[9] Griffith A.K. and Nichols N.K. Accounting for model error in data assimilation using adjoint methods, in M. Berz, C. Bischof, G. Corliss and A. Greiwank (eds.), Computational Differentiation: Techniques, Applications and Tools, SIAM, Philadelphia, 1996, pp. 195204.

[10] Griffith A.K., Martin M.J. and Nichols N.K. Techniques for treating systematic model error in 3D and 4D data assimilation, in Proceedings of the Third WMO Int. Symposium on Assimilation of Observations in Meteorology and Oceanography, World Meteorological Organization, 2000, WWRP Report Series No. 2, WMO/TD - No. 986, pp. 9-12.

[11] Le Dimet F.-X., Shutyaev V. On deterministic error analysis in variational data assimilation. Nonlinear Processes in Geophysics, 2005, v.12, p. 481-490. 
[12] Le Dimet F.-X., Shutyaev V.P., and Gejadze I. On optimal solution error in variational data assimilation: theoretical aspects. Russ. J. Numer. Anal. Math. Modelling, 2006, v.21, no. $2,139-152$.

[13] Le Dimet F.X., Talagrand O. Variational algorithms for analysis and assimilation of meteorological observations: theoretical aspects. Tellus, 1986, v.38A, pp.97-110.

[14] Lions J.L. Contrôle optimal des systèmes gouvernés par des équations aux dérivées partielles. - Paris: Dunod, 1968.

[15] Lorenc A.C. Analysis methods for numerical weather prediction, Q. J. Roy. Meteor. Soc., 1986, v.112, pp.1177-1194.

[16] Marchuk G.I., Agoshkov V.I., Shutyaev V.P. Adjoint Equations and Perturbation Algorithms in Nonlinear Problems. - New York: CRC Press Inc, 1996.

[17] Navon I.M. Practical and theoretical aspects of adjoint parameter estimation and identifiability in meteorology and oceanography. Dynamics of Atmospheres and Oceans, 1997, v.27, pp.55-79.

[18] Sasaki Y. Some basic formalism in numerical variational analysis. Month. Wea. Rev., 1970, v.98, no.12, pp.875-883.

[19] Shutyaev V.P., Le Dimet F.-X., Gejadze I.Yu. A posteriori error covariances in variational data assimilation. Russ. J. Numer. Anal. Math. Modelling, 2009, v.24 (2), pp.161-169.

[20] Stuart A.M. Inverse problems: a Bayesian perspective. Acta Numerica, 2010, v.19, pp $451-559$.

[21] Tarantola A. Inverse Problems Theory: Methods for Data Fitting and Model Parameter Estimation. - New York: Elsevier, 1987.

[22] Thacker W.C. The role of the Hessian matrix in fitting models to measurements. J. Geophys. Res., 1989, v.94, no.C5, pp.6177-6196.

[23] Vidard P.A. Vers une prise en compte de lerreur modèles en assimilation de données 4Dvariationnelle. Application à un modèle réaliste d'océan. Thèse de doctorat, Université Joseph Fourier (Grenoble), December 2001.

[24] Vidard P. A., Blayo E., Le Dimet F.-X. and Piacentini A. 4D variational data analysis with imperfect model. Flow. Turb. Comb., 2000, v.65, 489-504.

[25] Vidard P.A., Piacentini A., Le Dimet F.-X. Variational data analysis with control of the forecast bias. Tellus A, 2004, v.56 (3), pp.177-188. 